\title{
BULGARIAN MSDF R\&D NATIONAL PROGRAM AND ACTIVITIES
}

\author{
Emil SEMERDJIEV and Tzvetan SEMERDJIEV
}

\section{Introduction}

The Bulgarian National MSDF R\&D program was established more than twenty years ago. Bulgarian electronics and Bulgarian military industry were specialized in development and serial manufacturing of sea and river radars. Driven by increasing demands for better quality, faster production and minimum costs, MSDF R\&D projects have been constantly developed by research institutes and laboratories of Bulgarian industry and Bulgarian Academy of Science. The Bulgarian military branch has been especially interested in keeping abreast of the latest advances in this important field to ensure the highest standards and quality for military electronic systems modernization and upgrade. A number of young men have been educated in leading Soviet and East European military academies, civil technical universities and research centers, obtaining fundamental qualification. Since the mid 80's, the increased capabilities of computer performance provided a reliable base for direct implementation of scientific methods, previously considered as too expensive from computational point of view. Since then it was realized that the MSDF theory is an independent scientific branch requiring special attention and significant scientific efforts. Following this direction, a Multiple Sensors Data Fusion Department was founded in 1988 at the Bulgarian Academy of Sciences, as R\&D Laboratory named "SIGNAL". The most of research activities in Bulgaria in MSDF have been redirected from the industrial research centers toward this new unit. The major part of research activities in Bulgaria in this field were concentrated and accomplished in it. The Laboratory specialized in solving scientific and real-world problems involving signal, image and other type of data processing, with focus on the Bulgarian industry. It has become a leader in the design, development and evaluation of algorithms and application software for radar data processing and tracking systems. A number of $\mathrm{R} \& \mathrm{D}$ product implementations, mainly in the field of multiple target tracking 
algorithms (MTT) and related to it fields of simulation, guidance and control, have been accomplished:

- MTT algorithms and software implemented in meteorological radars (in serial manufacturing until 1990);

- MTT algorithms and software implemented in a parallel processor system for coastal radars (in serial manufacturing since 1990);

- mathematical models of signal and data processing systems for Complex Radar CAD systems (in use since 1988).

- Specialized Mathematical Library for radar data processing systems design and performance evaluation (in use since 1989);

- mathematical models and application software for Radar Simulators (in use since 1988)

- $\quad$ algorithms and software for unmanned ships collision avoidance systems.

A considerable scientific knowledge and experience has been accumulated in MSDF and especially in MTT during its eight-year history. In all these years, constant efforts for transition from defense-developed technologies to civilian government and commercial markets have been made, too.

\section{The Current Strategy}

The economical situation in Bulgaria after the Currency Board implementation two years ago became stabile and the success of the reforms is obvious. During this time it has been recognized that the further association of Bulgaria to the Western political, financial, and especially industrial, transport and military structures requires experts familiar with cutting-edge technologies (such as MSDF) capable to transfer knowledge and experience in Bulgaria and to participate in joint projects. That is the reason the general strategy of the most of research centers in Bulgaria has changed. The number and staff of the institutes and laboratories in industry and Bulgarian Academy of Science has been minimized. The strategy of their professional existence has been changed, too. A serious revision of the purpose and directions of the research activities has been made.

In the area of MSDF, the following directions of activity have been recognized as basic:

- preservation of existing scientific knowledge in the field of MSDF and accumulation of new one on a level that keeps the ability of nation to renovate technologically its industry; 
- provision of experts in such rapidly changing (in technological sense) fields as transportation, military armaments and other fields related to the MSDF technologies;

- transfer of new technologies in the mentioned fields supporting the process of transition of the Bulgarian industry towards western standards and structures.

- education of new generation highly qualified young experts and specialists for the needs of Bulgarian industry, administration and private business.

Applying these considerations in practice, some important steps have been accomplished:

- the laboratory has been reorganized and associated as a department named "Mathematical Methods for Sensor Data Processing" at the Central Laboratory for Parallel Processing at the Bulgarian Academy of Science, to provide a strong connection with researchers working in the field of mathematics (mathematical statistics and Monte Carlo methods) and computer science (parallel and distributed computer architectures);

- the research activities have been concentrated (but not restricted) in the field of MTT;

- $\quad$ some education courses, concerning the implementation of MSDF theory and practice in Air-Traffic Control, Strategic Leadership and Decision Making, DoD Science, High-Technologies \& Innovations Management have been developed from the department's staff and are in progress in Technical University in Sofia, Sofia's Business University, Rakovski War College and other schools;

- $\quad$ another area of activity is the participation in international research projects. Having experience in this field, based on successfully accomplished three R\&D projects in MTT field for foreign customers (ELTA Ltd. - Israel Aircraft Industry), the Department management considers this area as strategic.

\section{The Team}

In 1999 the department personnel consists of 13 researchers: two full professors with D.Sc. degrees, two associate professors and eight senior researchers with Ph.D. degrees. All specialists have broad knowledge and experience in:

- application of theoretic and systems approaches for solving the problems in tracking radars;

- developing and applying effective sensor data processing approaches and methods;

- base physical properties of large number of sensors and observed (air, sea and land) moving objects; 
- fields of scenario generation and use of real data for performance testing, and in the operator-machine interfaces testing \& evaluation and training.

- With classical scientific training and knowledge of advanced computer tools, software design and state-of-the-art technologies, the team provides the best solutions to customer problems.

\section{MSDF Research \& Development}

The field of MTT is an area where future joint fruitful collaboration is promising. Our achievements in this field relate mainly to tracking methods, algorithms and software development.

Generally speaking, the observed dynamic objects in our research studies are considered as hybrid stochastic systems. Such systems are characterized by continuous state and discrete set of unknown control, statistical or other parameters. A number of algorithms overcoming different kinds of uncertainty about the observed systems behavior and the ambiguity in the measurement sources, have been developed and their performance has been evaluated. These algorithms can be separated in five groups:

1. Recursive Pseudo-Bayesian algorithms estimating the hybrid state of a single object, based on the multiple model (MM) approach.

2. Recursive Pseudo-Bayesian algorithms associating measurements originating from multiple objects.

3. Data association algorithms using batch-processing techniques.

4. Recursive data classification \& association algorithms using attribute measurement data.

5. Multisource data association algorithms.

The algorithms from the first group include Interactive MM algorithms (IMM) ${ }^{1-10}$ with fixed structure $(\mathrm{FS})^{1-3}$ or with variable structure $(\mathrm{VS})^{4-10}$ and Generalized Pseudo-Bayesian algorithms (GPB). ${ }^{14}$ Recently, the FS algorithms are considered well studied, but we have succeeded in developing precise models of some commonly observed dynamic systems, improving their overall performance, as well as the performance of the VS algorithms. ${ }^{1-3,6,7}$ The development of different adaptive mechanisms, providing on-line adjustment of the unknown parameters in the IMM VS algorithms is a second perspective R\&D direction. ${ }^{4-10}$

The "bootstrap" (BS) approach is another promising hybrid state estimation tool, alternative to the above mentioned ones. Implementing the MM approach in it, a BSIMM algorithm was developed. ${ }^{11,12}$ It processes in real time an immense number of 
simulated data to identify and refine the $p d f$ of the observed system state. The algorithm demonstrates good estimation accuracy.

Our further efforts in the above mentioned fields are directed mainly towards generalization of the developed methods and their application in fault detection, robotics and other fields. ${ }^{6,32}$

The second group of algorithms comprises versions of the Probabilistic Data Association (PDA) approach and of the Multiple Hypotheses Tracking (MHT) algorithm. The developed PDA-IMM and BS-IMM-PDA versions ${ }^{13,21}$ demonstrate significant stability in dense clutter environments.

The developed versions of an object-oriented MHT algorithm are the core algorithms of the second group. ${ }^{14-21}$ Applying the MM approach, ${ }^{14,15}$ the Hough transform (HT), ${ }^{17,18}$ applying some heuristic techniques, ${ }^{15}$ and incorporating information about measurement features ${ }^{19,20}$ an overall improvement of MHT algorithm performance has been achieved. The results have been implemented in software CAD package, evaluating the algorithm performance through Monte Carlo simulations (see figures $1-4)$.

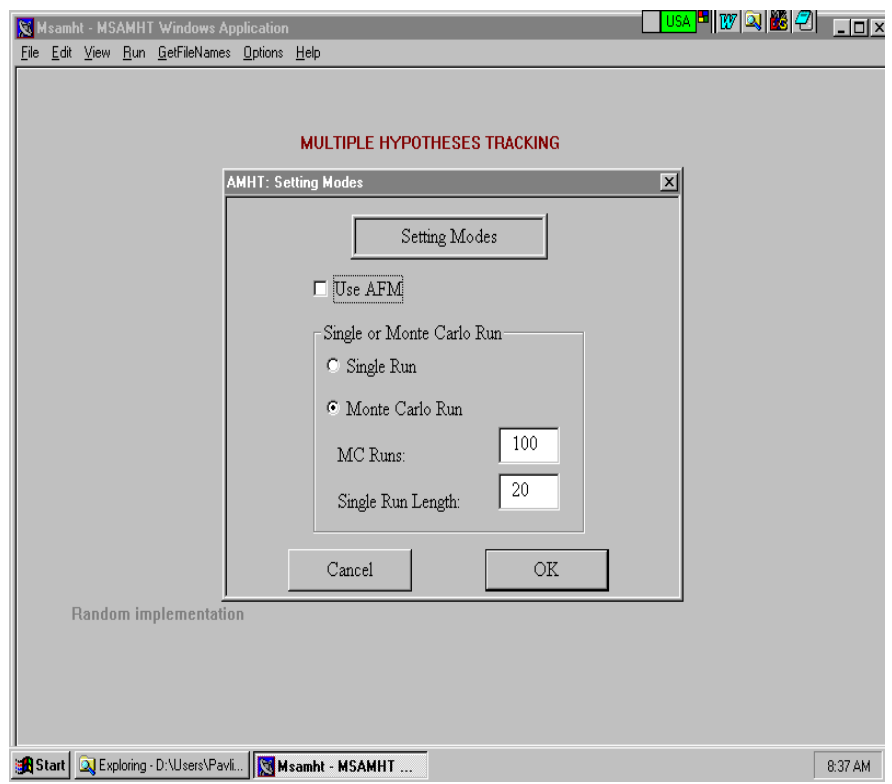

Figure 1: Dialog Setting Box 


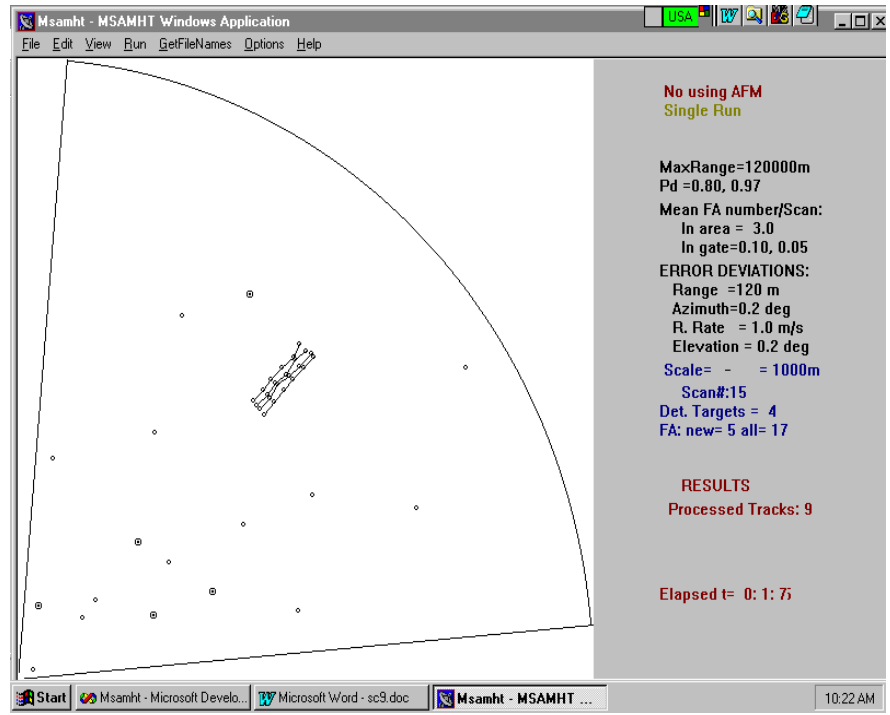

Figure 2: Graphical results visualization in a Single mode

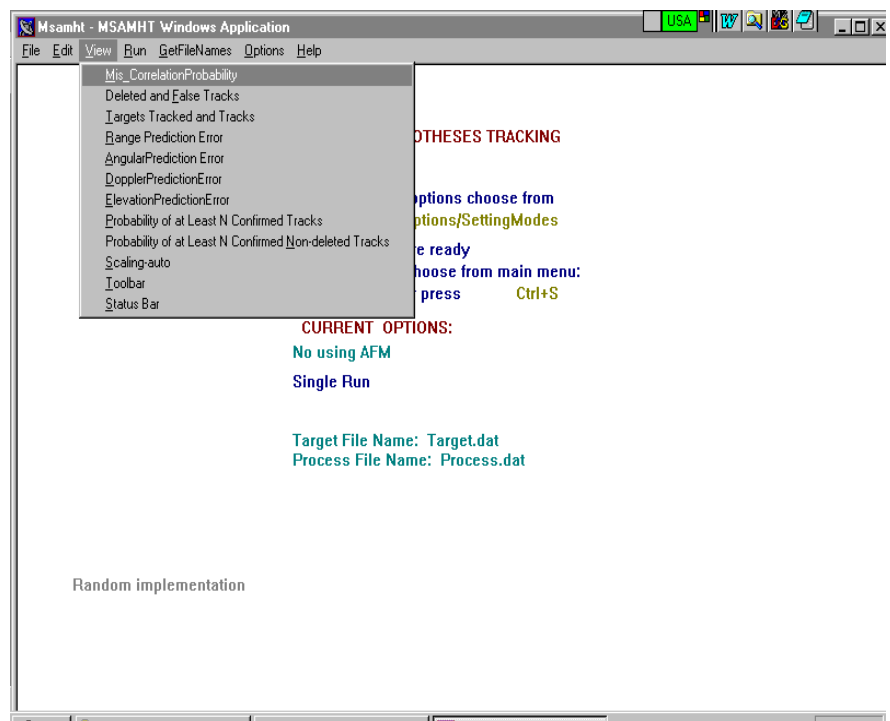

Figure 3: Measures of performance - the Control menu 


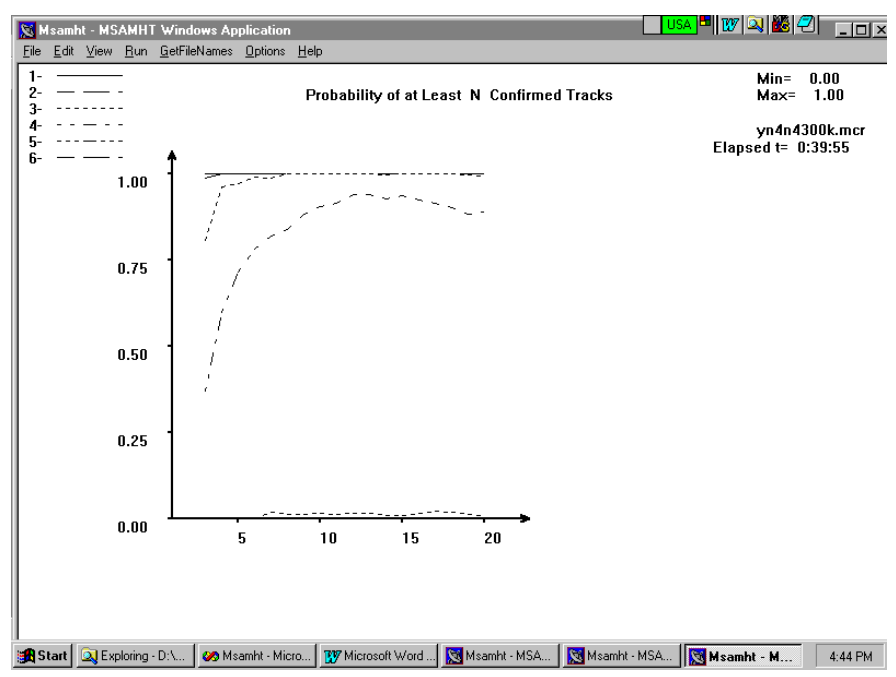

Figure 4: Monte Carlo graphical results visualization

The mentioned algorithms demonstrate high estimation accuracy in the hard case of maneuvering targets; significant clutter resistance; better data association and reduced computational load. ${ }^{15,18-20}$ The development of a software package implementing online all these MHT algorithms versions will be accomplished in the near future.

The proposed batch-processing data association algorithms from the third group are based on the application of HT as a track initiator and false alarm filter.17,18,24,25 We have solved problems concerning the identification of the connection between the base parameters of the HT algorithm, the sensor's parameters and the customer requirements related to track initiator performance. Further development of these algorithms is expected in their extension as adaptive track detectors and adaptive trackers.

The recursive data classification \& association algorithms using attribute measurement data from the fourth group are based on the combined application of evidence reasoning theory and fuzzy logic. In this way, some hard data association problems have been theoretically overcome: the problem of conflicting evidence, the presence of initial full ignorance in the arriving data, the problem of conflicting assignments etc. The proposed algorithms provide reliable decisions about the targets identity and affiliation. ${ }^{27-30}$ The application of the mentioned combined evidence reasoning theory with fuzzy logic approach instead the Bayesian one and is considered another promising area of investigation. 
The last group of algorithms concerns the multisource data association problem. It has been considered mostly with the practical aim to find a multisensor track initiation algorithm, resolving the synchronization problem and the combinatorial explosion arising in realistic multitarget cluttered environment. The development of multisensor MHT algorithm is another research direction that we intend to study in a greater detail. $^{25}$

A constant attention in most of the papers is paid to the problem of algorithm performance evaluation. The Monte Carlo simulation approach has been applied as a main tool to compute standard measures of performance in standard test scenarios. ${ }^{1,4,10,13,15,18-21}$

And finally, the problem of practical implementation of MTT algorithms is tightly connected with the required computational load. The inherent parallelism of some of the considered algorithms has been explored and the computational cost has been estimated. ${ }^{31-33}$

\section{Conclusion}

The brief examination of our strategy and achievements presented in this paper confirms our will to strengthen the relations with the international MSDF science community. Having in mind the emerging process of improving the industrial and financial situation in Bulgaria, we find that currently the R\&D joint projects are the most promising opportunity for fruitful cooperation.

\section{Acknowledgement}

The work on this paper was partially supported by the Bulgarian National Science Fund under contract No. I-801/98.

\section{References}

1. Semerdjiev, E.A., L.S. Mihaylova, and Tz.A. Semerdjiev, "Manoevring Ship Model Identification and IMM Tracking Algorithm Design," in Proc. of Intern. Conf. on Multisource-Multisensor Information Fusion - FUSION'98 (Las Vegas, Nevada: July 6-9, 1998), Vol.2, 968-973.

2. Angelova, D.S., V.P. Jilkov, and Tz.A. Semerdjiev, "Tracking Manoeuvring Aircraft by IMM Filters," Compte rendus de l'Academie Bulgare des Sciences 49, 12 (1996), 33-36. 
3. Semerdjiev, E.A. and V.G. Bogdanova, "Nonlinear Model and IMM Tracking Algorithm for Sea Radars," 40 Intern. Wissentschaftl. Kolloq. (Ilmenau, Germany: 1995), vol. 1, 140-145.

4. Semerdjiev, E.A. and L.S. Mihaylova, "Adaptive IMM Algorithm for Manoevring Ship Tracking," Proc. of Intern. Conf. on Multisource-Multisensor Inf. Fusion - FUSION'98 (Las Vegas, Nevada: July 6-9, 1998), 974-979.

5. Jilkov, V.P., D.S. Angelova, and Tz. Semerdjiev, "Mode-Set Adaptive IMM for Maneuvring Target Tracking,” IEEE Trans. AES 35 (1999), 343-350.

6. Mihaylova, L.S., Semerdjiev, E.A., and X. Rong-Li, "Detection and Localization of Faults in System Dynamics by IMM Estimator," In CD-ROM Proc. of the $2^{\text {nd }}$ Intern. Conf. on Multisource-Multisensor Information Fusion (Fusion' 99), Paper No. C-023, also in Conference Proceedings (Sunnyvale, California: 6-8 July, 1999), 937-943.

7. Semerdjiev, E.A., L.S. Mihaylova, L., and X. Rong-Li, "An Adaptive IMM Filter for Aircraft Tracking," In CD-ROM Proc. of the $2^{\text {nd }}$ Intern. Conf. on MultisourceMultisensor Information Fusion (Fusion'99), Paper No. C-064, also in Conference Proceedings (Sunnyvale, California: 6-8 July 1999), 770-777.

8. Jilkov, V.P., L.S. Mihaylova, and X. Rong-Li, "An Alternative IMM Filter for Benchmark Tracking Problem," Proc. of 1st Intern. Conf. on Multisource-Multisensor Inform. Fusion-FUSION' 98 (Las Vegas, Nevada: July 1998), vol. 2, 924-929.

9. Madjarov, N. and L.S. Mihaylova, “A Multiple-Model Adaptive Kalman Filter Algorithm for Continuous Systems," Comptes rendus de l'Academie Bulgare des Sciences 51, 9-10 (Sofia, Bulgaria: 1999), 49-53.

10. Jilkov, V.P. and D.S. Angelova, "Performance Evaluation and Comparison of Variable Structure Multiple-Model Algorithms for Tracking Maneuvring Radar Targets," in Proc. of $26^{\text {th }}$ Europ. Microwave Conf. (Prague, Czech Republic: 1996), 332-336.

11. Semerdjiev, Tz.A., V.P. Jilkov, and D.S. Angelova, "Target Tracking Using Monte Carlo Simulation," in Proc. of the IMACS Seminar on Monte Carlo Methods, Universite Libre de Bruxelle, 1-3 April 1997; published in Mathematics and Computers in Simulation (1998), 441-447.

12. Jilkov, V.P., D.S. Angelova, and Tz.A. Semerdjiev, "Filtering of Hybrid Systems: Bootstrap versus IMM," Proc. of European Conf. on Circuit Theory and Design (Budapest: 1997), Vol. 2, 873-878.

13. Angelova, D., Tz.A. Semerdjiev, V.P. Jilkov, and E.A. Semerdjiev, "Application of a Monte Carlo Method for Tracking Maneuvering Target in Clutter," in Proc. of the Second IMACS Seminar on Monte-Carlo Methods, pp. 61, Varna, 7-11 June, 1999 (accepted in Mathematics and Computers in Simulation).

14. Jilkov, V.P., D.S. Angelova and Tz.A. Semerdjiev, "Multiple Hypothesis Tracking Using GPB Filtering," Compte rendus de l'Academie Bulgare des Sciences 49, 12 (1996), 3336.

15. Jilkov, V.P., D.S. Angelova, and Tz.A. Semerdjiev, "Performance Evaluation of a Multiple Hypothesis Tracking Algorithm," Compte rendus de l'Academie Bulgare des Sciences 49, 12 (1996), 37-40.

16. Bojilov, L.V., "An Extension of an Algorithm for Multiple Hypotheses Tracking," Comptes rendus de l'Academie Bulgare des Sciences 50, 2 (1997), 45-48. 
17. Semerdjiev E.A., K.M. Alexieff, E.N. Djerassi, and Tz.A. Semerdjiev, "Multiple Hypothesis Tracking Algorithm Using Hough Transform," Compte rendus de l'Academie Bulgare des Sciences 49, 4 (1996), 37-40.

18. Semerdjiev E.A., K.M. Alexieff, E.N. Djerassi, and V.G. Bogdanova, "Performance Evaluation of a Multiple Hypothesis Tracking Algorithm Using Hough Transform," Compte rendus de l'Academie Bulgare des Sciences 49, 4 (1996), 51-54.

19. Jilkov V.P. and Tz.A. Semerdjiev, "Multiple Hypothesis Tracking Using Amplitude Feature Measurements," Compte rendus de l'Academie Bulgare des Sciences 49, 12 (1996), 41-44.

20. Semerdjiev, E.A., A.P. Tchamova, and P.D. Konstantinova, "Multiple Hypothesis Tracking Using Demster-Shafer's Attribute Class Estimation," Comptes rendus de l'Academie Bulgare des Sciences 50, 7 (1997), 53-56.

21. Jilkov, V.P., "Square-Root PDA Filtering," in Proc. Intern. Conf. on Mathematical Modelling and Scientific Computations (Sofia: DATECS Publishing, 1993), 67-72.

22. Jilkov, V.P., D. S. Angelova, and Tz.A. Semerdjiev, "State Estimation of a Nonlinear Dynamic System by Parallel Algorithms," in Proc. of EUROMECH'96 (1996), 215-218.

23. Semerdjiev, Tz., V.P. Jilkov, and D.S. Angelova, "Parallel Adaptive Algorithm for Dynamic Object State Filtering," Compte rendus de l'Academie Bulgare des Sciences 47, 5 (1994), 33-36.

24. Semerdjiev, E.A., "About the Application of the Hough Transform for Radar Track Detection," Comptes rendus de l'Academie Bulgare des Sciences 49, 9 (1996), 49-52.

25. Semerdjiev E., K.Alexiev and L.Bojilov, "Multiple Sensor Data Assosiation Using Hough Transform for Track Initiation," Proc. of the First International Conference on Multisource-Multisensor Information. Fusion (Las Vegas, Nevada: July 6-9, 1998), Vol.2, 980-985.

26. Semerdjiev, E., and A. Tchamova, "An Approach for a Fast Reduction of the Full Ignorance in the Target Identification Process," Proc. of the First International Conference on Multisource-Multisensor Information Fusion (Las Vegas, Nevada: July 69, 1998), Vol.2, 986-991.

27. Tchamova A.P., "Evidence Reasoning Theory with Application to the Identity Estimation and Data Association Systems," Mathematics and Computers in Simulation 43 (Netherlands: Elsevier Science, 1997), 139-142.

28. Tchamova A.P., "Evidence Reasoning Logic and Fuzzy Set Theory: Combined Method for Resolving Dempster's Rule Indefiniteness in a Case of Conflicting Evidence," Proc. of 4th European Congress on Intelligent Techniques and Soft Computing (Aachen, Germany: 1996), Vol. 1, 135-137.

29. Tchamova A.P., "Evidence Reasoning Logic and Fuzzy Set Theory: Combined Method for Resolving Conflict Assignments in Attributes Data Association Systems," IEEE Workshop on Nonlinear Signal and Image Processing, vol. II (Halkidiki, Greece, June 1995), 1011-1014.

30. Tchamova A.P., "Resolving Conflict Assignment in Attributes Data Association Systems," Comptes rendus de l'Academie Bulgare des Sciences 48,1 (1995), 57-60.

31. Konstentinova, P.D., E.N. Djerassi, V.G. Bogdanova, Parallelism in Multiple hypothesis Tracking Algorithm and Adaptation to Transputer Based Multiprocessor, 11th Intern. Conf. "Systems for Automation of Egineering and Research" (St. Konstantin, Bulgaria:1997), 81-85. 
32. Jilkov V. P., O. B. Manolov and D.S. Angelova, “A Parallel Algorithm for Mobile Robot Tracking," Proc. of the 14th IASTED International Conf. MODELLING, IDENTIFICATION AND CONTROL (Innsbruck, Austria: February 1996), 60-62.

33. Konstantinova P.D., E.A. Semerdjiev and A.P. Tchamova, "Performance Evaluation of Attribute Data Association Algorithm Using Parallel Processing," 10th Intern. Conf. on Systems for Automation of Engineering \& Research (SAER'96) (Varna, Bulgaria: 1996), 99-103.

TZVETAN ATANASOV SEMERDJIEV received D.Sc. degree in Rakovsky War College, Sofia, Bulgaria in 1986, Ph.D. and M.Sc. in Zhukovsky Air Force Engineering Academy, Moscow, Russia, 1973. He is member of IEEE, ICIF, AFCEA and the International Academy for Information Processing and Technologies, Moscow, Russia. Prof. Semerdjiev is member of the Editorial Board of "Information \& Security. An International Journal." E-mail: signal@bas.bg.

EMIL ATANASOV SEMERDJIEV (see page 68). 